\title{
POSSIBLE LINKS BETWEEN SUPERSONIC STELLAR WINDS AND THE ORIGIN OF COSMIC RAYS
}

T. Montmerle, M. Cassé, J. Paul

Section d'Astrophysique

Centre d'Etudes Nucléaires de Saclay, France

\section{I. $\quad$ COSMIC RAYS AND $\gamma$-RAYS IN THE GALAXY}

Cosmic rays (CR) are fast or relativistic protons and electrons (and nuclei), detected on the top of the terrestrial atmosphere. Except at the highest energies $(>101 \mathrm{keV})$ the Larmor radii of the particles in the galactic magnetic field are much smaller than the scale height of the interstellar gas, and the arriving particles have completely lost memory of their sources. Therefore, their origin is unknown, at least directly. But recent advances in $\gamma$-ray astronomy, and in shock acceleration mechanisms, have shed a radically new light on a old problem.

Gamma rays are high-energy photons which were successfully observed mainly by the American SAS-2 (30-200 MeV) and European COS-B (70 MeV-5 GeV) satellites. The $\gamma$-ray sky appears today as a thin line of diffuse emission, well correlated with the galactic disk (Mayer-Hasselwander et al. 1980), on which are superimposed a number of localized sources, having error boxes $\sim 1^{\circ}$ in radius (Swanenburg et al. 1980). Their typical flux is $z$ a few times $10^{-6} \mathrm{~cm}^{-2} \mathrm{~s}^{-1}$. Diffuse galactic $\gamma$-rays are produced mainly by : (a) collisions between 1-10 GeV protons and interstellar hydrogen (neutral, ionized or molecular), producing, via the strong interaction, neutral $\pi$ mesons, decaying into $2 \gamma$-rays (" $\pi^{\circ}$ decay"), and charged $\pi$ mesons, decaying in muons, neutrinos, and secondary electrons and positrons ; (b) collisions between $>50 \mathrm{MeV}$ electrons and interstellar hydrogen, emitting $\gamma$-rays by non-thermal bremsstrahlung (as do the secondary $\mathrm{e}^{-}$and $\mathrm{e}^{+}$accompanying $\pi^{\circ}$ decay). Both processes are thought to contribute about equally to the diffuse galactic flux locally. Also, both are proportional to the interstellar hydrogen density $n_{H}$ : as a result, in principle, once $n_{H}$ is known, one can deduce from $\gamma$-ray observations the corresponding CR density (see e.g. Stecker 1975).

\section{GALACTIC $\gamma$-RAY SOURCES}

On a large scale, the diffuse $\gamma$-ray emission is shown to be strongly correlated with galactic arms (Caraveo and Paul 1979), hence at least in part with their constituents (HII regions, hot stars, molecular clouds etc.) . On a smaller scale, 25 sources are now known. Their latitude distribution shows that their average distance from the Sun is $\langle d\rangle \sim 2-5 \mathrm{kpc}$, with a corresponding $\gamma$-ray luminosity $\left\langle L_{Y}>\sim 1034-1036\right.$ erg.s ${ }^{-1}$ (Swanenburg et al. 1980). It can be shown that up to half 
of the sources may possibly be identified with star forming regions : these include the $\rho$ Oph dark cloud ( $\sim 160 \mathrm{pc}$ ) (e.g. Cassé and Paul 1980), the Orion complex (d $\sim 500 \mathrm{pc}$ ) (Caraveo et al 1980) and SNOBs, i.e. supernova remnants physically linked with $\mathrm{OB}$ associations ( $\mathrm{d}>2 \mathrm{kpc}$ ) (Montmerle 1979). It can also be shown that a significant fraction of these sources must have a $\gamma$-ray emissivity higher than in the solar neighbourhood, hence a " built-in" CR source. In the $\rho$ Oph dark cloud, $\pi^{\circ}$ decay is probably the dominant mechanism, while $\gamma-$ ray spectral information on SNOBs is too poor to separate out non-thermal bremsstrahlung and $\pi^{\circ}$ decay. At any rate, this implies that one has to consider the possibility of in situ proton acceleration in these regions.

\section{PROTON ACCELERATION BY SHOCKS}

Aside from the conventional idea of particle acceleration by SN shocks (which includes recent theoretical developments, e.g., Blandford and Ostriker 1980), stellar winds (SW) have been recently proposed as possible additional particle accelerators, via the stationary shock which exists at the boundary with the surrounding medium (if the $\mathrm{SW}$ is supersonic). An explanation of $\gamma$-ray emission from the $\rho$ Oph cloud has recently been proposed along those lines, using winds from B and/or T Tau stars (Cassé and Paul 1980) (see discussion). A similar example may be provided by the Carina complex, on which we now focus (Montmerle et al. $1981 \mathrm{a}$, in preparation).

\section{a) Energetics}

The $\gamma$-ray source 2CG288-0 displays a striking positional coincidence with the Carina complex. It features most of the youngest stars known in the Galaxy (O3V) as well as 3 WR stars, and a SNR, for the existence of which these seems to be a somewhat controversial evidence (see details in Montmerle et al. 1981a). In view of this, the SW hypothesis should be tested as an alternative to the SN hypothesis. Let us compare the energetics, in the two cases, with $\mathrm{L}_{\gamma} \sim 5.10^{35}$ erg.s 1 at 2.7 kpc. (i) SN : using a conservative explosion energy $E_{\gamma} \gamma_{1051}$ ergs, one has, integrated over a typical SN lifetime of $<10^{5}$ years, $\mathrm{L}_{\gamma} \mathrm{dt} / \mathrm{E}_{0}<10^{5}$. (ii) $\mathrm{SW}$ : the total mechanical power $\mathrm{P}_{\mathrm{w}}$ available in the form of stellar winds in the Carina complex may be derived either from observation (WR stars, a few O stars; Conti and Garmany 1980, Barlow et al. 1980) or from empirical formulae (O stars ; Lamers et al. 1980), with the result $\mathrm{P}_{\mathrm{W}}(\mathrm{OB}) \sim 1038 \mathrm{ergs.s}^{-1}, \mathrm{P}_{\mathrm{W}}$ (WR) 2.1038 ergs.s ${ }^{-1}$. On the other hand, the peculiar object ${ }_{n}$ Car contributes a minimum of $\mathrm{P}_{\mathrm{w}}(n \mathrm{Car}) \sim 2.10^{38}$ ergs.s ${ }^{-1}$. Altogether, one has $\mathrm{L}_{\gamma} / \mathrm{P}_{\mathrm{w}}<10^{-3}$. Therefore, the energetics is as favourable in the case of SW as it is in the case of SN.

b) Acceleration efficiency

Now the shock-accelerated particles are scattered in the surrounding HII regions (NGC3372) by Alf vén scattering (e.g. Wentzel 1974), and are thus prevented to escape directly from it. It can be shown that, given the relatively high density of the ionized medium ( $\left.10^{2} \mathrm{~cm}^{-3}\right)$, the lifetime of protons with respect to inelastic collisions (which give rise mainly to $\pi^{\circ}$ decay $\gamma$-rays), is so short that they cannot leak out. One then calculates that the proton acceleration efficiency required from the $\mathrm{SN}$ or $\mathrm{SW}$ shock is $\sim 6 \%$, not an unreasonable requirement (but it can be much less in the case of a $S N$ shock if $E_{0} \gg 10^{51}$ ergs). 
Hence, in the Carina complex, SW shock acceleration may provide a viable alternative to $\mathrm{SN}$ shock acceleration or electron bremsstrahlung if it turns out that there is no SNR present.

c) Other $\mathrm{OB}$ associations

In order to check whether the SW hypothesis is compatible with the $\gamma$-ray data also elsewhere in the Galaxy, we have applied the same procedure to $72 \mathrm{OB}$ associations (Humphreys 1978), using empirical laws for mass-loss when required (Montmerle et al. 1981a,b,; Cassé et al. 1981a). Table 1 lists the expected $\gamma$-ray surface brightness of the 4 brightest $\mathrm{OB}$ associations, normalized to that of the Carina complex. The WR stars bring a dominant contribution. The figures obtained are not inconsistent with the $\gamma$-ray data.

Table 1

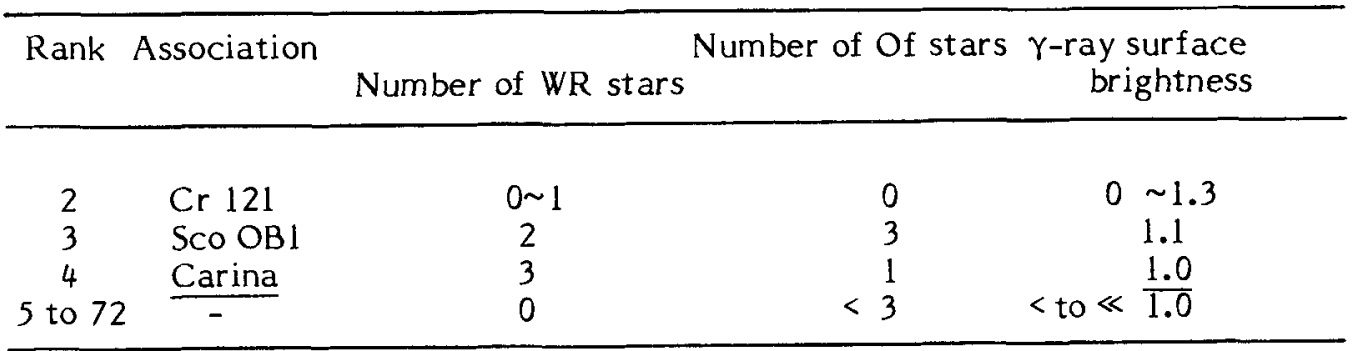

\section{A POSSIBLE CONSEQUENCE OF CR ACCELERATION BY SW SHOCKS}

Since WR stars play a dominant role in our mass-loss related complex, their peculiar composition is expected to show up, in CR nuclei, in a diluted form.

In this context, it is interesting to note that the ${ }^{22} \mathrm{Ne} /{ }^{20} \mathrm{Ne}$ at the $\mathrm{CR}$ source is $\sim 3$ times larger than in the solar system. Such an excess could be explained since the ${ }^{22} \mathrm{Ne}$ excess at the surface of a typical WC star (present as a result of ${ }^{14} \mathrm{~N}$ burning) is predicted to be $\sim 130$, relative to the solar system. Future observations of Neon in WR stars could thus prove or disprove this idea. The dilution, in the proportion $\sim 1 / 40$ of the ${ }^{22}$ Ne-rich component originating from WC stars, with the bulk of CR of "normal" composition, would lower the He and C excesses of the extra-component to a level compatible with the CR source abundances (Cassé et al. 1981b).

REFERENCES

Barlow, M.J. et al., 1980, preprint.

Blandford, R. D. and Ostriker, J.P., 1980, Ap.J. 237, 793.

Caraveo, P. and Paul, J., 1979, Astr. Ap. 75, $34 \overrightarrow{0 .}$

Caraveo, P. et al., 1980, Astr. Ap., in press.

Cassé, M. and Paul, J., 1980, Ap.J. 237, 236.

Cassé, M. et al., 1981a,b, Proc. IAU Symp. 94, ed. G. Setti et a1.,

Origin of Cosmic Rays, Reidel, Dordrecht, p. 35, 319. 
Conti, R.S. and Garmany, C.D., 1980, Ap.J. 238, 190.

Humphreys, R.M., 1978, Ap.J.Suppl. 38, 309.

Lamers, H.J.G.L.M. et al., 1980, Astr. Ap. 87, 68.

Mayer-Hasselwander, $\bar{H} . \bar{A}$. et al., 1980, Proc. 9th Texas Symp., Ann. N.Y.

Acad. Sci. 336, 211.

Montmerle, T., 1979, Ap.J. 231, 95.

Montmerle, T. et al., 1981a,b, Proc. IAU Symp. 94, ed. G. Setti et al Origin of Cosmic Rays, Reidel, Dordrecht, p. 317.

Stecker, F.W., 1975, in Origin of Cosmic Rays, Reidel, Dordrecht, p. 267. Swanenburg, B.N. et al., 1980, Ap.J. Letters, in press.

Wentzel, D.G., 1974, Ann. Rev. Astr. Ap. 12, 71.

\section{DISCUSSION}

CARRASCO: I probably have missed the main point in the case of the

@ Oph cloud. To my knowledge there are not o stars or early

$B$ supergiants associated with the cloud. However, there are wast numbers of $\mathrm{T}$ Tauri stars which present winds of smaller terminal velocities than

those of early type stars.

MONTMERLE: Correct. Cassi and Paul (1980) had used the B1III star

HD 147165, about 5 pc away, which on the basis of its spectral. type, was expected to release up $10^{36} \mathrm{erg} \mathrm{s}^{-1}$ in a wind, of which about $4 \%$ could interact with the cloud. But recent Copernicus observations by Snow show that this star sheds much less mass than expected. However, $\mathrm{T}$ Tau stars can do the job; in fact most of them are 1ikely buried inside the cloud. Their terminal velocities are instead smaller, but they are nevertheless supersonic, so that our proposed mechanism may still work. Our estimate of the minimum kinetic energy released is $10^{34} \mathrm{erg} \mathrm{s}^{-1}$ (Paul et al. 1980), still well above the observed X-ray luminosity of $8 \times 10^{32} \mathrm{erg} \mathrm{s}^{-1}$. 